Mainz

\title{
DIE STADTWERKE VON HATTUŠA
}

Als ich vor gut dreißig Jahren mein Studium der Indogermanistik bei Prof. Čop begann, riet er mir sofort am Anfang, mich dem Hethitischen zu widmen, wo noch so viel Neues zu erwarten wäre. Für diese Ermutigung bin ich ihm bis heute dankbar geblieben.

Beim Unterricht, der stets mitreißend und lebhaft war, galt seine besondere Begeisterung allen Sonderfällen, Cruces und hapax legomena.

Lange habe ich überlegt, womit ich dem verehrten Jubilar eine Freude bereiten könnte. Ich entschließ mich für einen Text, der mich schon seit Jahren beschäftigt und den ich trotz aller Mühe bis heute nicht übersetzen kann, teils wegen des schlechten Erhaltungszustandes, teils aber auch wegen einer auffällig hohen Zahl sonst unbelegter Wörter.

Es handelt sich um KUB 31.100 (CTH 275, Fragments de protocoles (ou de traités?)), eine Instruktion, die E. Laroche in die Nähe von KUB 13.2 (CTH 261, Instructions aux chefs de postes (bèl madgalti)) gestellt hat ${ }^{1}$. Obwohl eine zusammenhängende Übersetzung kaum möglich ist, verdient dieser Text, in einer möglichst zuverlässigen Umschrift vorgelegt zu werden, die vielleicht weitere Fortschritte in dessen Verständnis ermöglichen wird. Im "Boğazköy-Archiv" der Akademie der Wissenschaften und der Literatur in Mainz konnte ich meine alten Umschriftversuche anhand von Fotos überprüfen und einige zusätzliche Lesungen gewinnen.

Der Text enthält Vorschriften zum Bau und Instandhaltung von Gebäuden, Wasserversorgung, Instandhaltung von Brunnen und Zisternen, und zur Abfallentsorgung in der Hauptstadt Hattuša. Dies, sowie die Tatsache, da éin Beamter in der 2. Person Sg. - im Gegensatz zu der 2. Person Pl. der übrigen Instruktionstexte - angespochen wird, deutet auf eine Zugehörigkeit zur Textgruppe CTH 257, Instructions d'un Arnuwanda aux maires (hazannu) ${ }^{2}$. Besser wäre es wohl, von éinem HAZANNU zu sprechen $^{3}$. Inzwischen sieht man im "un Arnuwanda" den mittelhethitischen König Arnuwanda $\mathrm{I}^{4}$, obwohl alle Fragmente dieser Textgruppe jüngere Abschriften sind 5 .

1 E. Laroche, RHA IX/49, 1948-49, 12.

2 Ph. H.J. Houwink ten Cate, apud Schrijvend verleden. Documenten uit het oude Nabije Oosten vertaald en toegelicht, uitg. K.R. Veenhof. Leiden 1983, 176 Anm. 13.

3 Der HAZANNU ist bis jetzt nur in Hattuša belegt. H. Otten, FsKammenhuber 1983, 133-142, übersetzt den Titel mit "Bürgermeister", R.H. Beal, THeth 20, 1992, 437-442, mit "Governor of a Province Centered on a Major City" (im Gegensatz zu BEL MADGALTI "Governor of a Rural(?) Province"). 
Im Gegensatz zu den anderen Fragmenten dieser Textgruppe weist KUB 31.100 keine jüngere Zeichenformen aus, sondern nur die älteren, z.B. IK, AK, HAR, AH, E, URU. Das Zeichen ŠAR Rs. 8' endet mit nur einem senkrechten Keil, Rs. 16' scheint zwei zu haben, obwohl der letzte nicht ganz durchgezogen ist und in der Autographie gar nicht angedeutet wurde. Beachte auch die Ligatur kat+ta Rs. 10'.

Mit aller Vorsicht ließe sich die Niederschrift in den Anfang des 14. Jh. v. Chr. datieren, eine Bezeichnung "mittelhethitischer" Duktus ist nicht auszuschließen.

\begin{tabular}{|c|c|}
\hline Vs. 1 ' & $\mathrm{x}-\mathrm{x}-\mathrm{x}-m a k u-i t \mathrm{x}[$ \\
\hline 2 & $k u-i-e-e s ̌ n ~ n u ~ G I S ̌ s ~ I G-a n-n a ~ k[i-$ \\
\hline 3' & EGIR-pa ne-u-una-ah-ha-an e-eš-tu [ \\
\hline 4 & $a$-ša-an-du nam-ma še-er tu-e-x[ \\
\hline 5 , & $n u-u t-t a$ EGIR-an(-)ar-ha li-e x[ \\
\hline 6 & ${ }^{\mathrm{URU}} H a-a t-t u-s ̌ i-i a-[k a ́] n k u-e$ ku-e $\mathrm{X}[$ \\
\hline 7' & $h u-u-m a-a n-d a-a s ̌$ EGIR-an $a r-h b-u[t$ \\
\hline $8^{\prime}$ & ú-e-te-eš-kán-zi na-at EGIR-an[ \\
\hline 9' & bu-te-ek-ki-išs-kán-du úu-e-te-eš-ká[n-du \\
\hline $10^{\prime}$ & na-at $\breve{S} A$ EGIR.UD ${ }^{M I} u_{u}$-e-tum-mar e-eš-[tu \\
\hline $11^{\prime}$ & hu-u-te-ek-ki-ǐs-kán-du EGIR-an-da-m[a-at \\
\hline $12^{\prime}$ & ma-ak-nu-uš-kán- $\quad[d u$ \\
\hline $13^{\prime}$ & nam-ma ku-in $\mathrm{x}-\mathrm{x}$ tal-hi-in $\mathrm{x}[$ \\
\hline $14^{\prime}$ & 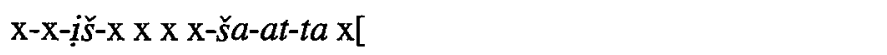 \\
\hline $15^{\prime}$ & $n u-z a$ tal-h $[i(-)$ o o $]$ bu-u-ma-an-ti-ina $Q A-T A M-M A \times[$ \\
\hline $16^{\prime}$ & $\check{S} A \hat{E}^{\mathrm{URU}}$ [o o o]x ku-i-e-eš BÀD-eš-na-aš a-ra-ah-z[é- \\
\hline $17^{\prime}$ & nu-uš tal-ḩa-ụ[-una-an-z]i zi-in-ni nam-ma IŠ-TU KÁ.GAL x] \\
\hline $18^{\prime}$ & $k u-i-e-e s ̌ s[00] \times$ a-pu-u-uš-ša ú-e-te nu-uš-kán $\times[$ \\
\hline $19^{\prime}$ & $I \check{S}$-TU KÁ.GAL x x x I-NA É ${ }^{\mathrm{URU}}$ Ha-la-ap-ša-an an[-da \\
\hline $20^{\prime}$ & 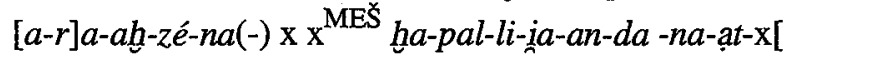 \\
\hline $21{ }^{\prime}$ & [o o]x huu-u-mạ-ạn $\quad z i-i n-\quad[n i]$ \\
\hline $22^{\prime}$ & {$\left[\begin{array}{lll}0 & 0 & 0\end{array}\right] \times$ x x x-te-eš-ki-ši iš-ki-iš-ki-mạ-ạt $\times[$} \\
\hline $233^{\prime}$ & 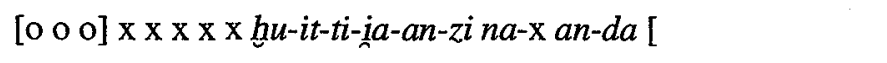 \\
\hline 24 & {$\left[\begin{array}{llll}0 & 0 & 0 & 0\end{array}\right] \times x \times\left[\begin{array}{lll}0 & 0 & 0\end{array}\right]$ hu-u-ru-te-eš-kán-zi(-)x[ } \\
\hline 25 ' & {$\left[\begin{array}{llllllll}0 & 0 & 0 & 0 & 0 & 0 & 0 & 0\end{array}\right] \times \underline{h} u-i t-t i-i a-u-\underline{n} a-a[n(-)$} \\
\hline $26^{\prime}$ & {$[000000000000-a] n-z i z i-i n-n i[$} \\
\hline $27^{\prime}$ & {$[00000000000000000] \times 1$} \\
\hline
\end{tabular}

5 J. Klinger und E. Neu, Hethitica 10, 1990, 145. 


\begin{tabular}{|c|c|c|}
\hline \multirow{2}{*}{ Rs. } & \multirow{2}{*}{$\begin{array}{l}1 \\
2^{\prime}\end{array}$} & \multirow{2}{*}{ 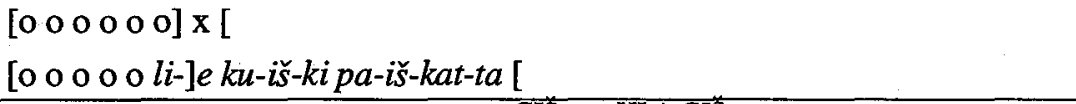 } \\
\hline & & \\
\hline & $3^{\prime}$ & 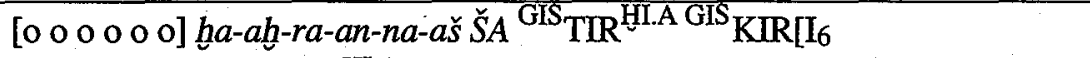 \\
\hline & $4^{\prime}$ & [o o o o o o 0 -]na-aš PU' $\mathrm{UU}^{\mathrm{HI} . \mathrm{A}}-a \check{s} k u$-ut-ta-aš EGIR-an(-)ša-ra-a[ \\
\hline & $5^{\prime}$ & [o o o o o o o]x-ri-na-ịa še-er I-NA HUR.SAG x-x-x [ \\
\hline & $6^{\prime}$ & 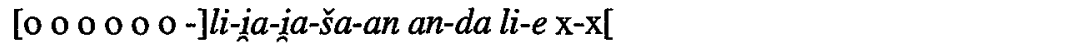 \\
\hline & 7 & [0 o o] $\mathrm{x}-d u-s ̌ a-k a ́ n$ an-da li-e pí-ěs-ši-iš-x[ \\
\hline & $8^{\prime}$ & [nu-za] ka-ma-ar-šu-una-aš ud-da-ni-i me-ek-ki na-ab-šar-x[ \\
\hline & 9' & 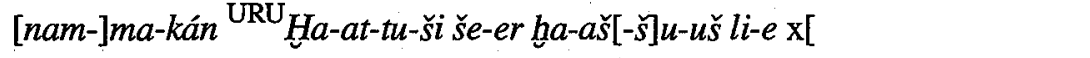 \\
\hline & $10^{\prime}$ & [n]a-aš-ta [b]a-aš-šu-uš kat-ta šal-la-a-i hu-ǔšši-li-pá[t \\
\hline & 11 ' & nam-ma ku-i-e-eš ku-i-e-eš ku-e-lu-una-ni-eš še-er É-x[ \\
\hline & $12^{\prime}$ & $k u-i-e-e s ̌$ nam-ma ku-i-e-eš ku-una-pí nu-uš hुu-u-ma-an-du[-uš \\
\hline & 13' & una-na-al-li-iš-kán-du iš-tal-ki-iš-kán- $d[u]$ \\
\hline & 14 & 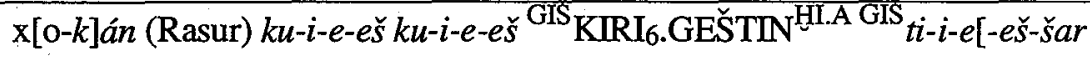 \\
\hline & $15^{\prime}$ & 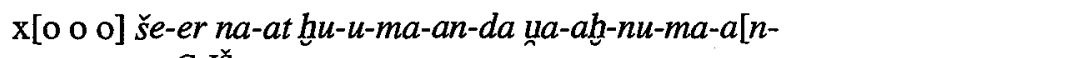 \\
\hline & $16^{\prime}$ & $\left.\mathrm{x}\left[\begin{array}{lllll}0 & 0 & 0 & 0 & 0\end{array}\right]^{\mathrm{G}}\right]_{t i-i-e-e s ̌-s ̌ a r ~ h u-u-m a-a n ~ h u-u-u ̣ r[-}$ \\
\hline & $17^{\prime}$ & $x\left[\begin{array}{lllllllll}0 & 0 & 0 & 0 & 0 & 0 & 0 & 0\end{array}\right]$ ši-iš-šu-u-ri-ia-u-una-an-z$[i$ \\
\hline & 18 ' & 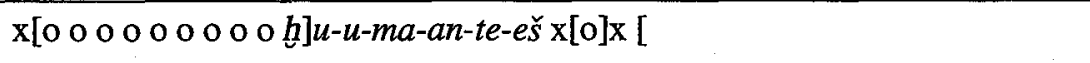 \\
\hline & $19^{\prime}$ & 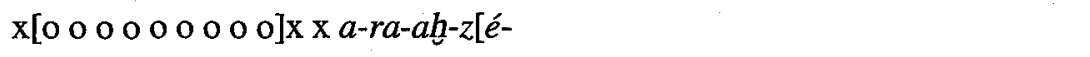 \\
\hline & $20^{\prime}$ & 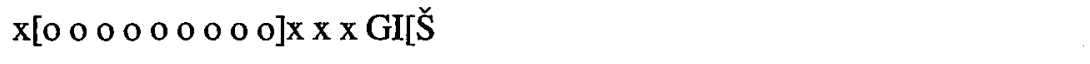 \\
\hline & $21^{\prime}$ & $x\left[\begin{array}{llll}0 & 0 & 0 & 0\end{array}\right] \times x[$ \\
\hline & $22{ }^{\prime}$ & $\mathrm{x}[$ \\
\hline & $23^{\prime}$ & $\mathrm{x}^{\mathrm{HI} . \mathrm{A}-T I M}-m a[$ \\
\hline
\end{tabular}

\section{PHILOLOGISCHER KOMMENTAR}

Vs. 2'f. Zur Instandsetzung von Türen und ähnlichen Vorrichtungen vgl. KUB 31.86 Vs. II 14f. "Die Tore, Posternen, Treppeneingänge, Stadtbefestigungen sollen mit Tür und Riegel versehen sein, nichts darf abgenutzt werden".

Vs. 5' EGIR-an(-)ar-ha ist univerbiert.

Vs. 7" "Kümmere dich um alles!", s. HW" A 201; vgl. fast synonym [...EGI]R-an ar-h̆u-ut nu-kán IGIHI.A-una har-ak 706/v Z. 4'.

Vs. 9', 11' hutekkkiške-, nach J. Puhvel, HED 3, 1991, 417, Iterativ zu hutk-, Variante von hatk-; eine Bedeutung “dicht machen" würde im Kontext von Baumaßnahmen gut passen.

Vs. 10'-12' s. CHD s.v. maknu- 'Let it be a permanent building. Let them keep ...-ing it [es soll dicht gemacht werden], and afterwards let them increase (or accumu- 
late) [...]". Wahrscheinlich ist das Objekt von maknu- hier unetummar "das Gebäude", das sorgfältig ausgeführt werden soll und bei Bedarf auch erweitert oder angebaut werden soll. Vs. 10' benutzte A. Ünal, JCS 40, 1988, 97-106, als Titel für einen Aufsatz über die Rolle des hethitischen Architekten ("You should build for eternity"); vgl. auch id., Belleten LII/205, 1989, 1470 ("Let it be a construction for eternity").

Vs. 13', 15', 17' talhi- (Subst.), talhai- (Verb): die ersten beiden Belege sind unergiebig, aber es scheint sich weiterhin um Bauvorschriften zu handeln. Vs. 16'f. könnte man ungefähr mit "Die äußeren Befestigungsmauern des Hauses der Stadt... sollst du fertig talhai-" wiedergeben. Die Infinitiv-Form ist zwar keineswegs gesichert, aber durch Spuren, Raum und Verbindung mit Imp.Sg.2 zinni wohl sehr wahrscheinlich. Einen Bau der äußeren Befestigungsmauer würde man durch Auskleidung mit behauenen Steinen abschließen. Das erinnert an einen ähnlichen Vorgang, wo ein Wallgraben mit Stein gepflastert wird, nämlich KUB 31.86 Vs. II 12'. In der Umschrift von E. von Schuler, Dienstanw. 43 lautet [X] ša-ra-a ǏS-TU ZÁx?: X-ri ha-a-an$d u$. In seiner Rezension schlägt E. Laroche, RHA XV/61, 1957, 127 die Lesung [nu] IŠ-TU NA 4 [?] : [p]a-tal-ha-a-an-du. Er schreibt weiter: "Devant patalhandu, on a soit le double Glossenkeil, soit la fin d'un idéogramme effacé après $\mathrm{NA}_{4}$. Pour patalha(i)-, dénominatif de patalha-, cf. HW 166: "chausser, cheviller"?". Sein Vorschlag wurde von A. Goetze, JCS 13, 1959, 69f. bestätigt. Vgl. auch R.H. Beal, THeth 20, 1992, 242 Anm. 920: “... let them secure (:patalhāndu [coll.])". Ich habe die Stelle am Foto nochmals kollationiert und bin zum folgenden Ergebnis gekommen: die Rasur fängt unmittelbar nach $I \check{S}$ - $T U$ an und reicht bis einschließlich RUTAL. Über die Rasur wurde dann $\mathrm{NA}_{4}$ und RITAL geschrieben, alles dazwischen ist getilgt. Demnach wäre also $\mathrm{NA}_{4}$ (Rasur) tal-ha-a-an-du zu lesen, Die Verbindung zur Wortsippe patalhawäre damit hinfällig, obwohl der Kontext noch weiterhin eine Deutung wie etwa "(mit Steinen) auslegen, bedecken, pflastern, auskleiden" verlangt.

Vs. 17' Imp.Sg.2 zinni kann nun bei N. Oettinger, Stammbildung 1979, 312 das Paradigma vin zinne- "beendigen, fertig werden mit etwas" ergänzen.

Vs. 19' INA É URU Halap=šan wohl als Syntagma am Satzanfang zu verstehen. Handelt es sich um eine Institution in Hattuša? Zu ähnlichen regionalen Vertretungen in der Haupstadt vgl. R.H. Beal, THeth 20, 1992, 53f. mit Anm. 202. Vielleicht ist É URU [Ha-la-a]p auch Vs. 16' zu ergänzen.

Vs' 20' hapalliiant- "beschädigt(?)", im Anschluß an ein, bei J. Puhvel, HED 3, 1991, 116 (s.v. hapallasai-), noch mit einem Asteriskus versehenen Verb hapallai-.

Vs. 22' iškiški=ma=at Iter.Imp.Sg.2 zu išk(iịa)- "salben, bestreichen", am häufgisten durchgeführt mit Feinöl, Honig, Fett oder Bier; beachte jedoch KBo 21.22, 43 AN.BAR-at ǐ̌-ki-ia mit A. Kammenhuber, HW2 A 366 s.v. arzila/i- "mit Eisen ist er gesalbt").

Vs. 24' huruteške- “(um)stoßen(?)” o.ä., s. J. Puhvel, HED 3, 1991, 408 s.v. hurutil-. Vielleicht auch hu-u-ru-te-ěs-ki-ši Vs. 22' (vor iškiške-), aber äußerst unsicher. 
Rs. 3' nicht hahrannǎ̌š=a (so J. Puhvel, HED 3, 1991, 6), sondern - $a \check{S} \breve{S} A$ “der geharkten [Felder?], der Wälder, der Gärt[en".

Rs. 4' erwähnt "Wände der Brunnen"; EGIR-an(-)ša-ra-a univerbiert.

Rs. 5' Die Spuren hinter HUR.SAG könnten ta-a-ha[ gelesen werden. Kann es sich dabei wirklich um den gutbekannten Berg Taha handeln, der ja zwischen Zipalanta und Ankuwa, also etwa $60 \mathrm{~km}$ südöstlich von Hुattuša, lag? Wir haben keine Angaben, wie groß der Verwaltungsbezirk der Haupstadt war.

Rs. 6' vielleicht [...lu-ú-]li-ĩa-ina-ša-an zu ergänzen.

Rs. 7' das erste Zeichen nach dem Bruch weist zwei senkrechte Keile auf. Eine mögliche Lesung wäre -i]š-. In diesem Absatz ist die Rede von Brunnen und Wasserbecken ( $l u l i-$ ), die dicht und sauber gehalten werden sollen. Diese Vorrichtungen standen in den Feldern, Wäldern und Gärten, vielleicht auch als Bewässerungsanlagen. Rs. 6'f. also etwa "[Schütte] kein [...] in den Becken, werfe keine...-išta - hinein!"

Rs. 8'-10' 'In der Angelegenheit der Fäkalien seid sehr vorsichtig! Ferner, die Asche darf man nicht oben in Hattuša [ausschütten], die Asche soll man ausschließlich (-pát) unten in der großen Grube [ausschütten]". Diese Stelle wurde mehrfach behandelt und verschiedentlich interpretiert.

Rs. 8' kamaršuunaš uddanĩ "cacandi causa" E. Kronasser, EHS 1963, 302, vgl. E. Neu, GsKronasser 1982, 134.

Statt des in den Instruktionen üblichen Partizips nahhant- "vorsichtig" (CHD s.v. $n a h(h)-b .1$ ') steht hier eine Form von nahšariia- in gleicher Bedeutung, die in CHD für diese Wortsippe nicht verbucht ist.

Rs. 12'f. haššuš Pl.Akk. "Asche”, s. J. Puhvel, HED 3, 1991, 210, ähnlich auch Ph.JH.J. Houwink ten Cate, op.cit. (s.Anm.2), 176 ("haarden", "(oude) oventjes"). Die Bedeutung "Asche” ist bei Puhvel, loc. cit., durch Parallelen gestützt und scheint sinnvoll im Kontext zu sein. Fäkalien und Asche unterliegen besonderen Entsorgungsvorschriften. Da dieser Absatz innerhalb von Vorschriften, die Wasserversorgung betreffen, eingebettet ist, muß es sich um Maßnahmen gegen Wasserverunreinigung handeln.

Die zweite Deutung, die von H.G. Güterbock, Oriens 10, 1957, 353, stammt, ist zwar theoretisch nicht auszuschließen, da ein Verbum ja fehlt, ist aber sachlich und inhaltlich nicht haltbar. Güterbock sah in haššuš (Sg.Nom.) sie syllabische Schreibung für LUGAL-uš "König", aufgenommen von J. Friedrich, HW 2. Erg. 12, F. Gentili Pieri, AttiAccTosc. 47, 1982, 27f., und J.J.S. Weitenberg, U-Stämme, 1984, 436 Anm. 375.

Rs. 10' huššili- "Grube”, s. J. Puhvel, HED 3, 1991, 409f., vgl. auch N. BoysanDietrich, THeth 12, 1987, 7 Anm. 2. Eine Bedeutung "Abort, Latrine" erübrigt sich, da die Ablage der Fäkalien hier nicht vorgeschrieben ist, nur die Asche muß ausdrücklich in die große Grube in der Unterstadt ausgeschüttet werden.

Rs. 11'-13' "Ferner, welche kueluuana-Behälter oben im Haus [...] und welche auch sonst wo sind, sollen allesamt ge-unanallai-t und geglättet werden!", s. CHD 3, 388, s.v., namma 5.f. 
Rs. 13' unanalliške- hapax legomenon, bezeichnet einen technischen Vorgang, der dem des ištalkiške- "glatt, eben machen" ähnlich ist. Letzters ist auch in KUB 31.86 II 17' mit Dupl. KUB 31.89 II 6' im Zusammenhang mit Bautätigkeiten belegt, vgl. J. Puhvel, HED 2, 1984, 451.

Man kann aus diesem Zusammenhang schließen, daß die kueluunana-Behälter gebaute, wasserdicht gemachte Zisternen waren, die sowohl innerbalb von Gebäuden, als auch sonst wo im Stadtgebiet verstreut waren.

Rs. 14', 16' GIŠ tīeššar und GIŠTIR HI.A Rs. 3' werden normalerweise mit "Wald" übersetzt. Hier gehört es zusammen mit Weingärten, Gärten und bebauten Feldern (?, hahrannaš Rs. 5') zu den kultivierten und bewässerten Anbauflächen. Kann es sich eher um Obstgärten handeln?

Rs. 17’ ̌̌iššuriīauñanzi Inf. "bewässern”, -iš- ist über getilgtes -eš- geschrieben.

Der hier behandelte Teil der Instruktionen beschränkt sich also auf zwei Aufgabenbereiche des "Bürgermeisters" von Hुattuša. Während die Vorderseite Baumaßnahmen für einen sauber ausgeführten und sicheren Bau vorschreibt, befaßt sich die Rückseite mit der Pflege der Wasservorrichtungen, nämlich von Brunnen und Zisternen, mit dem Schutz vor Verschmutzung und mit Bewässerungsanlagen.

[Korekturzusatz: Siehe auch die Umschrift und Übersetzung des Textes von A. Ünal, Ritual Purity Versus Physical Impurity in Hittite Anatolia, Apud Uluslararas1 1. Hititoloji Kongresi Bildirileri (19-21) Temmuz 1990) Çorum (o.J.), S. 196f., 215f.]

\section{Povzetek \\ KOMUNALA V HATTUŠI}

Slabo ohranjeni tekst KUB 31.100 sodi v žanr instrukcij, pravilnikov za visoke državne uradnike. Predpisi zadevajo varno in trdno zidavo stavb ter njihovo vzdrževanje na eni strani, na drugi pa skrb za vodnjake in cisterne, namakanje vinogradov, vrtov in sadovnjakov, ter za čistočo vode. Tudi za odlaganje odpadkov je poskrbljeno. Predpisi veljajo za hetitsko glavno mesto, Hattušo, zato so najbrž naslovljeni na njenega župana. Na podlagi paleografije lahko ugotovimo, da je bil tekst zapisan ob koncu 15. ali začetku 14. stol. pr.Kr. 